\title{
CURVATURE-ADAPTED REAL HYPERSURFACES IN QUATERNIONIC SPACE FORMS
}

\author{
Toshiaki Adachi and Sadahiro Maeda
}

\begin{abstract}
In this paper we study geodesics on curvature-adapted real hypersurfaces in nonflat quaternionic space forms. By observing the extrinsic shape of geodesics on these hypersurfaces we characterize them in the class of real hypersurfaces. We also investigate the length spectrum of geodesic spheres, which are the simplest curvature-adapted real hypersurfaces, in non-flat quaternionic space forms.
\end{abstract}

\section{Introduction}

The aim of this paper is to study curvature-adapted real hypersurfaces in quaternionic space forms from the viewpoint of the extrinsic shape of geodesics on these hypersurfaces. A hypersurface $M$ of a Riemannian manifold $\tilde{M}$ is called curvature-adapted if the normal Jacobi operator $K$ and the shape operator $A$ of $M$ with respect to a unit normal vector field $\mathscr{N}$ are simultaneously diagonalizable; $K \circ A=A \circ K$. Here the normal Jacobi operator $K: T M \rightarrow T M$ of $M$ with respect to $\mathscr{N}$ is defined by $K(\cdot)=\tilde{R}(\cdot, \mathscr{N}) \mathscr{N}$ with the curvature tensor $\tilde{R}$ of $\tilde{M}$. In his paper [B], J. B. Berndt completely classified curvature-adapted real hypersurfaces $M^{4 n-1}$ in a quaternionic projective space $\boldsymbol{H} P^{n}$ of quaternionic dimension $n(\geqq 2)$. They are locally congruent to tubes around the canonically embedded quaternionic projective spaces of quaternionic dimension $k(0 \leqq k \leqq$ $n-1)$ or tubes around the canonically embedded complex projective space of complex dimension $n$. He also classified curvature-adapted real hypersurfaces $M$ in a quaternionic hyperbolic space $\boldsymbol{H} H^{n}$ of quaternionic dimension $n(\geqq 2)$ under the additional condition that all principal curvatures of $M$ in $\boldsymbol{H} H^{n}$ are constant. They are locally congruent to either a horosphere, tubes around canonically embedded quaternionic hyperbolic spaces of quaternionic dimension $k(0 \leqq k \leqq$

2000 Mathematics Subject Classification: primary 53C22, secondary 53C40.

The first author partially supported by Grant-in-Aid for Scientific Research (C) (No. 11640073), Ministry of Education, Science, Sports and Culture.

The second author partially supported by Grant-in-Aid for Scientific Research (C) (No. 11640079), Ministry of Education, Science, Sports and Culture.

Received April 28, 2000; revised October 25, 2000. 
$n-1)$ or tubes around the canonically embedded complex hyperbolic space of complex dimension $n$.

In the first half of this paper we characterize these curvature-adapted real hypersurfaces in non-flat quaternionic space forms, which are quaternionic projective spaces and quaternionic hyperbolic spaces, by the extrinsic shape of geodesics. It is known that non-flat quaternionic space forms do not admit real hypersurfaces all of whose geodesics are circles in them. We consider a weaker condition. When the ambient manifold $\tilde{M}$ is a quaternionic Kähler manifold we have a natural orthogonal decomposition $\mathscr{D} \oplus \mathscr{D}^{\perp}$ of the tangent bundle $T M$ of a real hypersurface $M$ : The subbundle $\mathscr{D}$ is the maximal subbundle of $T M$ which is invariant by the quaternionic structure of $\tilde{M}$. We characterize these curvatureadapted real hypersurfaces in non-flat quaternionic space forms by the existence of an orthonormal basis of $\mathscr{D}_{x}$ at each point $x$ such that the extrinsic shape of geodesics of those directions are circles of positive curvature in the ambient manifolds.

When the ambient manifold is a non-flat complex space form, curvatureadapted real hypersurfaces are nothing but real hypersurfaces whose structure vector field $J \mathscr{N}$ is a principal curvature vector field. In preceding papers [AKM] and $[\mathrm{CM}]$ these real hypersurfaces with constant principal curvatures are characterized by a similar condition in our results Theorems 3.1 and 3.2. Our theorems in this paper can be regarded as quaternionic versions of preceding results.

In the second half of this paper we study the extrinsic shape of all geodesics on geodesic spheres in a non-flat quaternionic space form, which are the simplest examples in the class of real hypersurfaces. By direct computation we can see that each geodesic on these geodesic spheres is regarded as a helix of order 4 which lies on a totally geodesic complex space form of complex dimension 2 . As a consequence we clarify properties on lengths of all closed geodesics on these geodesic spheres.

\section{Modeled real hypersurfaces in quaternionic space forms}

A quaternionic Kähler structure $\mathscr{J}$ on a Riemannian manifold $\tilde{M}$ of real dimension $4 n$ is a rank 3 vector subbundle of the bundle of endmorphisms of the tangent bundle $T \tilde{M}$ with the following properties:

1) For each point $\tilde{x} \in \tilde{M}$ there is an open neighborhood $\tilde{G}$ of $\tilde{x}$ in $\tilde{M}$ and sections $J_{1}, J_{2}, J_{3}$ of the restriction $\left.\mathscr{J}\right|_{\tilde{G}}$ over $\tilde{G}$ such that

i) each $J_{i}$ is an almost Hermitian structure on $\tilde{G}$, that is, $J_{i}^{2}=-$ id and $\left\langle J_{i} \tilde{X}, \tilde{Y}\right\rangle+\left\langle\tilde{X}, J_{i} \tilde{Y}\right\rangle=0$ for all vector fields $\tilde{X}$ and $\tilde{Y}$ on $\tilde{G}$, where $\langle$,$\rangle is the Riemannian metric of \tilde{M}$.

ii) $J_{i} J_{i+1}=J_{i+2}=-J_{i+1} J_{i}(i \bmod 3)$ for $i=1,2,3$.

2) $\tilde{\nabla}_{\tilde{X}} J$ is a section of $\mathscr{J}$ holds for each vector field $\tilde{X}$ on $\tilde{M}$ and section $J$ of the bundle $\mathscr{J}$, where $\tilde{\nabla}$ denotes the Riemannian connection of $\tilde{M}$. This triple $\left\{J_{1}, J_{2}, J_{3}\right\}$ is called a canonical local basis of $\mathscr{J}$. For each canonical local basis of quaternionic structure, there exist three 1-forms $q_{1}, q_{2}$ and $q_{3}$ on $\tilde{G}$ satisfying 


$$
\tilde{\nabla}_{\tilde{X}} J_{i}=q_{i+2}(\tilde{X}) J_{i+1}-q_{i+1}(\tilde{X}) J_{i+2} \quad(i \bmod 3)
$$

for each vector field $\tilde{X}$ on $\tilde{G}$ and $i=1,2,3$.

Let $M$ be a real hypersurface of a quaternionic Kähler manifold $\tilde{M}$, which is a Riemannian manifold equipped with a quaternionic Kähler structure $\mathscr{J}$, and $\mathscr{N}$ be a unit normal vector field of $M$. We denote by $\mathscr{D}$ the maximal subbundle of $T M$ which is invariant by $\mathscr{J}$ : At a point $x \in M$ the subspace $\mathscr{D}_{x}$ is the maximal subspace of $T_{x} M$ with the property that $J v \in \mathscr{D}_{x}$ for each $v \in \mathscr{D}_{x}$ and $J \in \mathscr{J}_{x}$. Let $\mathscr{D}^{\perp}$ denote the orthogonal complement of $\mathscr{D}$ in $T M$. It is a rank 3 vector subbundle of $T M$. By using a canonical local basis $\left\{J_{1}, J_{2}, J_{3}\right\}$ of $\mathscr{J}$ over an open subset $\tilde{G}$ of $\tilde{M}$ containing $x$, we find that $\mathscr{D}_{x}^{\perp}$ is the real linear subspace of $T_{x} M$ spanned by $J_{1} \mathscr{N}, J_{2} \mathscr{N}, J_{3} \mathscr{N}$. On an open set $G=\tilde{G} \cap M$ we set $\xi_{i}=-J_{i} \mathscr{N}$ and define $\phi_{i}: T M \rightarrow T M$ by $\phi_{i}=\left.\pi \circ J_{i}\right|_{T M}$ for $i=1,2,3$, where $\pi:\left.T \tilde{M}\right|_{M} \rightarrow$ $T M$ is the canonical projection. Then the following identities hold on $G$ for $i=$ $1,2,3$ :

$$
\begin{aligned}
& \phi_{i} \xi_{i}=0, \quad \phi_{i} \xi_{i+1}=\xi_{i+2}, \quad \phi_{i} \xi_{i+2}=-\xi_{i+1}, \\
& \left.\phi_{i} \circ \phi_{i+1}\right|_{\mathscr{D}}=\left.\phi_{i+2}\right|_{\mathscr{D}}=-\left.\phi_{i+1} \circ \phi_{i}\right|_{\mathscr{D}} \quad(i \bmod 3) .
\end{aligned}
$$

Let $\nabla$ denote the Riemannian connection of $M$ and $A$ the shape operator of $M$ with respect to $\mathscr{N}$. It follows from (2.1) and the Weingarten formula for a real hypersurface $M$ that

$$
\nabla_{X} \xi_{i}=q_{i+2}(X) \xi_{i+1}-q_{i+1}(X) \xi_{i+2}+\phi_{i} A X
$$

for each vector field $X$ on $G$.

We call a connected quaternionic Kähler manifold $\tilde{M}$ a quaternionic space form of quaternionic sectional curvature $c(\in \boldsymbol{R})$ if the Riemannian sectional curvature of $\tilde{M}$ is equal to $c$ for all tangent 2-planes spanned by $u \in T_{\tilde{x}} \tilde{M}$ and $J u$ with $J \in \mathscr{F}_{\tilde{x}}$ at each point $\tilde{x} \in \tilde{M}$. The standard models of quaternionic space forms are a quaternionic projective space $\boldsymbol{H} P^{n}(c)$ of quaternionic sectional curvature $c(>0)$, a quaternionic Euclidean space $\boldsymbol{H}^{n}$ and a quaternionic hyperbolic space $\boldsymbol{H} H^{n}(c)$ of quaternionic sectional curvature $c(<0)$.

In his paper $[B]$, Berndt characterized curvature-adapted real hypersurfaces in quaternionic space forms. Following his results, when $M$ is a real hypersurface in a non-flat quaternionic space form, the following three conditions are equivalent each other:

(1) $M$ is curvature-adapted.

(2) The subbundle $\mathscr{D}$ is invariant by the shape operator of $M$.

(3) The subbundle $\mathscr{D}^{\perp}$ is invariant by the shape operator of $M$.

The above condition (3) guarantees the existence of an open and dense subset $\mathscr{U}_{0}$ of a curvature-adapted real hypersurface $M$ with the following property: For every $x \in \mathscr{U}_{0}$ there exists a canonical local basis $\left\{J_{1}, J_{2}, J_{3}\right\}$ of $\mathscr{J}$ on a neighborhood $\tilde{G}$ of $x$ in a non-flat quaternionic space form $\tilde{M}$ satisfying that $G(=\tilde{G} \cap M)$ $\subset \mathscr{U}_{0}$ and $\xi_{i}$ is a principal curvature vector of $M$ at each $x \in G$. Moreover, each of the principal curvatures $\alpha_{i}$ associated with $\xi_{i}$ is locally constant on $G$. 
THEOREM A ([B], cf. [MP]). (1) A connected curvature-adapted real hypersurface in a quaternionic projective space $\boldsymbol{H} P^{n}(4)(n \geqq 2)$ is locally congruent to one of the following real hypersurfaces:

$\left(\mathrm{P}_{1}\right)$ A tube $P_{1}^{k}(r)$ of some radius $r \in(0, \pi / 2)$ around the canonically (totally geodesic) embedded $\boldsymbol{H} P^{k}$ for some $k \in\{0, \ldots, n-1\}$.

$\left(\mathrm{P}_{2}\right)$ A tube $P_{2}(r)$ of some radius $r \in(0, \pi / 4)$ around the canonically (totally geodesic) embedded complex projective space $\boldsymbol{C} P^{n}$.

(2) A connected curvature-adapted real hypersurface with constant principal curvatures in a quaternionic hyperbolic space $\boldsymbol{H} H^{n}(-4)(n \geqq 2)$ is locally congruent to one of the following real hypersurfaces:

$\left(\mathrm{H}_{1}\right)$ A tube $H_{1}^{k}(r)$ of some radius $r \in(0, \infty)$ around the canonically (totally geodesic) embedded $\boldsymbol{H} H^{k}$ for some $k \in\{0, \ldots, n-1\}$.

$\left(\mathrm{H}_{2}\right)$ A tube $\mathrm{H}_{2}(r)$ of some radius $r \in(0, \infty)$ around the canonically (totally geodesic) embedded complex hyperbolic space $\mathrm{CH}^{n}$.

$\left(\mathrm{H}_{3}\right)$ A horosphere $\mathrm{H}_{3}$ in $\boldsymbol{H H}^{n}$.

(3) Conversely, each of these modeled real hypersurfaces is a curvatureadapted real hypersurface with constant principal curvatures in the ambient manifold.

We here list the principal curvatures and their multiplicities of these modeled real hypersurfaces.

\begin{tabular}{|c|c|c|c|c|c|}
\hline & $P_{1}^{k}(r)$ & $P_{2}(r)$ & $H_{1}^{k}(r)$ & $H_{2}(r)$ & $H_{3}$ \\
\hline$\lambda_{1}$ & $\cot r$ & $\cot r$ & $\operatorname{coth} r$ & $\operatorname{coth} r$ & 1 \\
\hline$\lambda_{2}$ & $-\tan r$ & $-\tan r$ & $\tanh r$ & $\tanh r$ & - \\
\hline$\mu_{1}$ & $2 \cot 2 r$ & $2 \cot 2 r$ & $2 \operatorname{coth} 2 r$ & $2 \operatorname{coth} 2 r$ & 2 \\
\hline$\mu_{2}$ & - & $-2 \tan 2 r$ & - & $2 \tanh 2 r$ & - \\
\hline$m\left(\lambda_{1}\right)$ & $4(n-k-1)$ & $2(n-1)$ & $4(n-k-1)$ & $2(n-1)$ & $4(n-1)$ \\
\hline$m\left(\lambda_{2}\right)$ & $4 k$ & $2(n-1)$ & $4 k$ & $2(n-1)$ & - \\
\hline$m\left(\mu_{1}\right)$ & 3 & 1 & 3 & 1 & 3 \\
\hline$m\left(\mu_{2}\right)$ & - & 2 & - & 2 & - \\
\hline
\end{tabular}

Here $\lambda_{j}$ is an eigenvalue of $\left.A\right|_{\mathscr{D}}$ and $\mu_{j}$ is that of $\left.A\right|_{\mathscr{D}^{\perp}}$, and $m(\cdot)$ denotes the multiplicity of corresponding eigenvalue.

For real hypersurfaces of type $\left(\mathrm{P}_{1}\right),\left(\mathrm{H}_{1}\right)$ and $\left(\mathrm{H}_{3}\right)$ in Theorem $\mathrm{A}$ we find the following properties hold.

Proposition 2.1 (cf. [P]). Every curvature-adapted real hypersurface $M$ of type $\left(\mathrm{P}_{1}\right),\left(\mathrm{H}_{1}\right)$ or $\left(\mathrm{H}_{3}\right)$ in the lists of Theorem $A$ satisfies the following: 
(1) The structure tensor $\phi_{i}$ and the shape operator $A$ of $M$ are commutative: $\phi_{i} A=A \phi_{i}(i=1,2,3)$.

(2) The covariant derivative of the shape operator A satisfies

$$
\left(\nabla_{X} A\right) Y=\mp \sum_{i=1}^{3}\left\{\left\langle\phi_{i} X, Y\right\rangle \xi_{i}+\left\langle\xi_{i}, Y\right\rangle \phi_{i} X\right\},
$$

where the double sign depends on the case that either the ambient space is $\boldsymbol{H} P^{n}(4)$ or $\boldsymbol{H} H^{n}(-4)$.

For real hypersurfaces of type $\left(\mathrm{P}_{1}\right)$ in $\boldsymbol{H} P^{n}$ this proposition is due to $[\mathrm{P}]$. For real hypersurfaces of type $\left(\mathrm{H}_{1}\right)$ or $\left(\mathrm{H}_{3}\right)$ in $\boldsymbol{H} H^{n}$ we can check these properties by the same argument as for Lemma 4.2 in [P] and Theorem 4.3 in [NR].

In the last stage of this section we write down the Codazzi equation for a curvature adapted real hypersurface in a quaternionic projective space $\boldsymbol{H} P^{n}(c)$ and a quaternionic hyperbolic space $\boldsymbol{H} H^{n}(c)$, which will be usefull in the following sections:

$$
\left(\nabla_{X} A\right) Y-\left(\nabla_{Y} A\right) X=\frac{c}{4} \sum_{i=1}^{3}\left\{\left\langle X, \xi_{i}\right\rangle \phi_{i} Y-\left\langle Y, \xi_{i}\right\rangle \phi_{i} X-2\left\langle\phi_{i} X, Y\right\rangle \xi_{i}\right\} .
$$

\section{Characterization of curvature-adapted real hypersurfaces}

A smooth curve $\gamma=\gamma(s)$ on a Riemannian manifold $N$ parametrized by its arclength $s$ is called a Frenet curve of proper order $d$ if there exist orthonormal frame fields $\left\{X_{1}=\dot{\gamma}, \ldots, X_{d}\right\}$ along $\gamma$ and positive functions $\kappa_{1}(s), \ldots, \kappa_{d-1}(s)$ which satisfy the following system of ordinary equations

$$
\nabla_{\dot{\gamma}} X_{j}(s)=-\kappa_{j-1}(s) X_{j-1}(s)+\kappa_{j}(s) X_{j+1}(s), \quad j=1, \ldots, d,
$$

where $X_{0} \equiv X_{d+1} \equiv 0$ and $\nabla_{\dot{\gamma}}$ denotes the covariant differentiation along $\gamma$ with respect to the Riemannian connection $\nabla$ of $N$. Equation (3.1) is called the Frenet formula for a Frenet curve $\gamma$. The functions $\kappa_{j}(s)(j=1, \ldots, d-1)$ and the orthonormal frame $\left\{X_{1}, \ldots, X_{d}\right\}$ are called the curvatures and the Frenet frame of $\gamma$, respectively. We call a smooth curve a helix of order $d$ when it is a Frenet curve of proper order $r(\leqq d)$ and all its curvatures are constant. A helix of order 1 is nothing but a geodesic. A helix of order 2 with the first curvature $\kappa_{1}=\kappa$ is called a circle of curvature $\kappa$. A smooth curve $\gamma$ parametrized by its arclength is a circle of curvature $\kappa$ if and only if it satisfies $\nabla_{\dot{\gamma}} \nabla_{\dot{\gamma}} \dot{\gamma}=-\kappa^{2} \dot{\gamma}$.

Let $l$ denote the isometric immersion of a real hypersurface $M$ into a non-flat quaternionic space form $\tilde{M}$. For a smooth curve $\gamma$ on $M$ we call the curve $l \circ \gamma$ on $\tilde{M}$ the extrinsic shape of $\gamma$. For simplicity we usually denote the curve $l \circ \gamma$ by $\gamma$. The aim of this section is to characterize curvature-adapted real hypersurfaces in non-flat quaternionic space forms in Theorem A by the extrinsic shape of some geodesics on these hypersurfaces. 
THEOREM 3-1. Let $M$ be a connected real hypersurface of a quaternionic projective space $\boldsymbol{H} P^{n}$. Then $M$ is locally congruent to a real hypersurface of type $\left(\mathrm{P}_{1}\right)$ or $\left(\mathrm{P}_{2}\right)$ if and only if at each point $x \in M$ there exists an orthonormal basis $\left\{v_{1}, \ldots, v_{4 n-4}\right\}$ of $\mathscr{D}_{x}$ such that all geodesics $\gamma_{j}=\gamma_{j}(s)$ on $M$ with $\gamma_{j}(0)=x$ and $\dot{\gamma}_{j}(0)=v_{j}(1 \leqq j \leqq 4 n-4)$ are circles of positive curvature in $\boldsymbol{H} P^{n}$.

THEOREM 3-2. Let $M$ be a connected real hypersurface of a quaternionic hyperbolic space $\boldsymbol{H H}^{n}$. Then $M$ is locally congruent to a real hypersurface of type $\left(\mathrm{H}_{1}\right),\left(\mathrm{H}_{2}\right)$ or $\left(\mathrm{H}_{3}\right)$ if and only if at each point $x \in M$ there exists an orthonormal basis $\left\{v_{1}, \ldots, v_{4 n-4}\right\}$ of $\mathscr{D}_{x}$ such that all geodesics $\gamma_{j}=\gamma_{j}(s)$ on $M$ with $\gamma_{j}(0)=x$ and $\dot{\gamma}_{j}(0)=v_{j}(1 \leqq j \leqq 4 n-4)$ are circles of positive curvature in $\boldsymbol{H}^{n}$.

We shall prove these theorems simultaneously. In this section, let $\tilde{M}$ denote a non-flat quaternionic space form. Namely, $\tilde{M}$ denotes either a quaternionic projective space or a quaternionic hyperbolic space. We denote by $\tilde{\nabla}$ the Riemannian connection of $\tilde{M}$. We use the following technical results due to Berndt $[\mathrm{B}]$ on curvature-adapted real hypersurfaces in a non-flat quaternionic space form.

Proposition B ([B]). Let $M$ be a curvature-adapted real hypersurface in a quaternionic space form of quaternionic sectional curvature $c(\neq 0)$. We choose a canonical local basis of $\mathscr{J}$ on $\tilde{G}$ such that each $\xi_{i}$ is a principal curvature vector; $A \xi_{i}=\alpha_{i} \xi_{i}$.

(1) At each point $x \in G=\tilde{G} \cap M$, for each principal curvature vector $v \in \mathscr{D}_{x}$ with $A v=\lambda v$,

$$
\left(2 \lambda-\alpha_{i}\right) A \phi_{i} v=\left(\lambda \alpha_{i}+\frac{c}{2}\right) \phi_{i} v
$$

holds for every $i=1,2,3$.

(2) $\alpha_{i}=\alpha_{j}$ or $q_{k} \mid \mathscr{D}_{x}=0$ for all distinct $i, j, k \in\{1,2,3\}$.

(3) If $\alpha_{i} \neq \alpha_{i+1}=\alpha_{i+2}$, then $q_{i+1}\left(\xi_{i}\right)=q_{i+2}\left(\xi_{i}\right)=0(i \bmod 3)$.

["Only if" part of the proof of Theorems 3.1 and 3.2.]

In the first place we prove the only if part. Let $M$ be a real hypersurface in a non-flat quaternionic space form $\tilde{M}$ satisfying the following condition $(*)$ :

At each point $x \in M$ there exists an orthonormal basis $\left\{v_{1}, \ldots, v_{4 n-4}\right\}$

$(*)$ of $\mathscr{D}_{x}$ such that all geodesics $\gamma_{j}=\gamma_{j}(s)$ on $M$ with $\gamma_{j}(0)=x$ and $\dot{\gamma}_{j}(0)=v_{j}(1 \leqq j \leqq 4 n-4)$ are circles of positive curvature in $\tilde{M}$.

By this condition the geodesic $\gamma_{j}$ whose initial vector is $v_{j}$ satisfies

$$
\tilde{\nabla}_{\dot{\gamma}_{j}}\left(\tilde{\nabla}_{\dot{\gamma}_{j}} \dot{\gamma}_{j}\right)=-\kappa_{j}^{2} \dot{\gamma}_{j}
$$

for some positive constant $\kappa_{j}$.

On the other hand, from the formulae of Gauss and Weingarten; 


$$
\tilde{\nabla}_{X} Z=\nabla_{X} Z+\langle A X, Z\rangle \mathscr{N} \text { and } \tilde{\nabla}_{X} \mathscr{N}=-A X
$$

we have

$$
\tilde{\nabla}_{\dot{\gamma}_{j}}\left(\tilde{\nabla}_{\dot{\gamma}_{j}} \dot{\gamma}_{j}\right)=\left\langle\left(\nabla_{\dot{\gamma}_{j}} A\right) \dot{\gamma}_{j}, \dot{\gamma}_{j}\right\rangle \mathscr{N}-\left\langle A \dot{\gamma}_{j}, \dot{\gamma}_{j}\right\rangle A \dot{\gamma}_{j} .
$$

By comparing the tangential components of the equalities (3.2) and (3.3) we find

$$
\left\langle A \dot{\gamma}_{j}(s), \dot{\gamma}_{j}(s)\right\rangle A \dot{\gamma}_{j}(s)=\kappa_{j}^{2} \dot{\gamma}_{j}(s),
$$

in particular, at $s=0$

$$
\left\langle A v_{j}, v_{j}\right\rangle A v_{j}=\kappa_{j}^{2} v_{j} .
$$

By noticing that $\kappa_{j} \neq 0$ we get

$$
A v_{j}=\kappa_{j} v_{j} \quad \text { or } \quad A v_{j}=-\kappa_{j} v_{j} \quad \text { for } j=1, \ldots, 4 n-4 .
$$

This implies that $\mathscr{D}_{x}$ is invariant by the shape operator $A$. Since $x \in M$ is arbitrary, we find that our real hypersurface $M$ is curvature-adapted to $\tilde{M}$. When $\tilde{M}$ is a quaternionic projective space, we obtain $M$ is locally congruent to a real hypersurface of type $\left(\mathrm{P}_{1}\right)$ or $\left(\mathrm{P}_{2}\right)$ by Berndt's classification.

What we have to do is to prove that when $\tilde{M}$ is a quarternionic hyperbolic space all principal curvatures of our curvature-adapted real hypersurface $M$ are constant on $M$. Consider an open and dense subset $\mathscr{U}$ of $M$ given by

$$
\mathscr{U}=\left\{x \in \mathscr{U}_{0} \mid \begin{array}{l}
\text { the multiplicity of each principal curvature of } M \\
\text { in } \boldsymbol{H} H^{n} \text { is constant on some neighborhood } \mathscr{V}_{x} \text { of } x
\end{array}\right\} .
$$

Then all principal curvatures are differentiable on $\mathscr{U}$. For each point $x \in \mathscr{U}$, if we choose sufficiently small neighborhood $\mathscr{V}_{x}$, we can choose principal curvature vectors to be smooth and the principal curvatures $\alpha_{i}(i=1,2,3)$ associated with $\xi_{i}$ are constant on this neighborhood.

At a fixed point $x \in \mathscr{U}$, we denote by $\lambda_{1}, \lambda_{2}, \ldots, \lambda_{g}$ the distinct principal curvatures at $x$, which can be extended to differentiable functions on $\mathscr{V}_{x}$. Since the principal curvatures associated with $\xi_{i}(i=1,2,3)$ are constant on $\mathscr{V}_{x}$, we shall prove that each $\lambda_{l}$ associated with $\left.A\right|_{\mathscr{D}}$ is constant on some neighborhood of $x$. By (3.4) there is $j_{l}$ such that either $\lambda_{l}(x)=\kappa_{j_{l}}$ or $\lambda_{l}(x)=-\kappa_{j_{l}}$ holds. We only treat the former case; $A v_{j_{l}}=\kappa_{j_{l}} v_{j_{l}}$. Since the geodesic $\gamma_{j_{l}}$ with the initial vector $v_{j_{l}} \in T_{x} M$ on $M$ is a circle of curvature $\kappa_{j_{l}}$ on $\tilde{M}$, we have $\lambda_{l}\left(\gamma_{j_{l}}(s)\right)=\kappa_{j_{l}}$ for each $s$, hence find $v_{j_{l}} \lambda_{l}=0$. As $A$ is symmetric, we also have

$$
\left\langle\left(\nabla_{v_{j_{l}}} A\right) v_{k}, v_{j_{l}}\right\rangle=\left\langle v_{k},\left(\nabla_{v_{j_{l}}} A\right) v_{j_{l}}\right\rangle, \quad 1 \leqq k \neq j_{l} \leqq 4 n-4 .
$$

In order to compute (3.5) easily, we extend the tangent vectors $v_{k}, v_{j_{l}} \in T_{x} M$ to vector fields $V_{k}, V_{j_{l}}$ on some sufficiently small neighborhood $\mathscr{W}_{x}\left(\subset \mathscr{V}_{x}\right)$ in the following manner: We denote by $W_{j l}$ the smooth vector field on $\mathscr{V}_{x}$ defined by parallel displacement of the vector $v_{j_{l}}$ along each geodesic through $x$. Although 
$W_{j_{l}}$ is not principal on $\mathscr{V}_{x}$ in general, we have $A W_{j_{l}}\left(\gamma_{j_{l}}(s)\right)=\kappa_{j_{l}} W_{j_{l}}\left(\gamma_{j_{l}}(s)\right)$. We define a vector field on $\mathscr{V}_{x}$ by

$$
U_{j_{l}}=\left(\prod_{\substack{1 \leqq m \leqq g \\ m \neq l}}\left(A-\lambda_{m} I\right)\right) W_{j_{l}} .
$$

Clearly, at each point $y \in \mathscr{V}_{x}$ the vector $U_{j_{l}}(y)$ is $\prod_{m \neq l}\left(\lambda_{l}-\lambda_{m}\right)$ times of the projection of $W_{j_{l}}(y)$ onto the eigenspace corresponding to $\lambda_{l}(y)$. Hence $A U_{j_{l}}=$ $\lambda_{l} U_{j_{l}}$ on $\mathscr{V}_{x}$. We choose a neighborhood $\mathscr{W}_{x}\left(\subset \mathscr{V}_{x}\right)$ of $x$ such that every $U_{j_{l}}$ does not vanish on it, and set $V_{j_{l}}=U_{j_{l}} /\left\|U_{j_{l}}\right\|$. Our construction guarantees that $A V_{j_{l}}=\lambda_{l} V_{j_{l}}$ on $\mathscr{W}_{x}$ and $V_{j_{l}}(x)=v_{j_{l}}$, and that the integral curve of $V_{j_{l}}$ through the point $x$ is a geodesic on $M$. In particular, we obtain $\left(\nabla_{V_{j_{l}}} V_{j_{l}}\right)(x)=0$. For $v_{k}$ we choose a section $V_{k}$ of $\left.\mathscr{D}\right|_{\mathscr{W}_{x}}$ with $V_{k}(x)=v_{k}$.

Since the Codazzi equation (2.5) implies $\left\langle\left(\nabla_{X} A\right) Y, Z\right\rangle=\left\langle\left(\nabla_{Y} A\right) X, Z\right\rangle$ for arbitrary sections $X, Y, Z$ of $\mathscr{D}$, we find at the point $x$

(The left hand side of $(3.5))=\left\langle\left(\nabla_{v_{k}} A\right) v_{j_{l}}, v_{j_{l}}\right\rangle$

$$
\begin{aligned}
& =\left\langle\left(\nabla_{V_{k}} A\right) V_{j_{l}}, V_{j_{l}}\right\rangle_{x} \\
& =\left\langle\left(V_{k} \lambda_{l}\right) V_{j_{l}}+\left(\lambda_{l} I-A\right) \nabla_{V_{k}} V_{j_{l}}, V_{j_{l}}\right\rangle_{x} \\
& =v_{k} \lambda_{l} .
\end{aligned}
$$

Similarly we get

$$
\begin{aligned}
\text { The right hand side of }(3.5)) & =\left\langle V_{k},\left(\nabla_{V_{j_{l}}} A\right) V_{j_{l}}\right\rangle_{x} \\
& =\left\langle V_{k}, \nabla_{V_{j_{l}}}\left(\lambda_{l} V_{j_{l}}\right)-A \nabla_{V_{j_{l}}} V_{j_{l}}\right\rangle_{x} \\
& =\left\langle v_{k},\left(v_{j_{l}} \lambda_{l}\right) v_{j_{l}}\right\rangle=0 .
\end{aligned}
$$

These imply that $X \lambda_{l}=0$ for every $X \in \mathscr{D}_{x}$.

Next, we shall prove $\xi_{i}(x) \lambda_{l}=0(i=1,2,3)$. We may suppose either one of the following conditions holds:

(I) $2 \lambda_{l}-\alpha_{i} \neq 0$ for every $i=1,2,3$ on some neighborhood of $x$,

(II) $2 \lambda_{l}-\alpha_{i} \equiv 0$ holds on a neighborhood of $x$ for some $i$, because the set of points $y \in \mathscr{U}$ with such a property is a dense open subset of $M$. Since $\alpha_{i}$ is constant on $\mathscr{V}_{x}$, we have only to study the case (I). In this case, we obtain from Proposition B

$$
A \phi_{i} V_{j_{l}}=\left(\frac{\alpha_{i} \lambda_{j_{l}}-2}{2 \lambda_{j_{l}}-\alpha_{i}}\right) \phi_{i} V_{j_{l}}
$$

on some neighborhood $\mathscr{W}_{x}^{\prime}\left(\subset \mathscr{W}_{x}\right)$ of $x$. This, together with Proposition B and (2.3), yields 


$$
\begin{aligned}
\left(\nabla_{\xi_{i}} A\right) & V_{j_{l}}-\left(\nabla_{V_{j_{l}}} A\right) \xi_{i} \\
= & \nabla_{\xi_{i}}\left(A V_{j_{l}}\right)-A \nabla_{\xi_{i}} V_{j_{l}}-\nabla_{V_{j_{l}}}\left(\alpha_{i} \xi_{i}\right)+A \nabla_{j_{j_{l}}} \xi_{i} \\
= & \nabla_{\xi_{i}}\left(\lambda_{l} V_{j_{l}}\right)-A \nabla_{\xi_{i}} V_{j_{l}}-\alpha_{i} \phi_{i} A V_{j_{l}}+A \phi_{i} A V_{j_{l}} \\
& \quad+q_{i+2}\left(V_{j_{l}}\right)\left(\alpha_{i+1}-\alpha_{i}\right) \xi_{i+1}-q_{i+1}\left(V_{j_{l}}\right)\left(\alpha_{i+2}-\alpha_{i}\right) \xi_{i+2} \\
= & \left(\xi_{i} \lambda_{l}\right) V_{j_{l}}+\left(\lambda_{l} I-A\right) \nabla_{\xi_{i}} V_{j_{l}}-\lambda_{l}\left(\alpha_{i}-\frac{\alpha_{i} \lambda_{l}-2}{2 \lambda_{l}-\alpha_{i}}\right) \phi_{i} V_{j_{l}} .
\end{aligned}
$$

On the other hand, (2.5) yields

$$
\left\langle\left(\nabla_{\xi_{i}} A\right) V_{j_{l}}-\left(\nabla_{V_{j_{l}}} A\right) \xi_{i}, V_{j_{l}}\right\rangle=0 .
$$

By combining these two equalities we find $\xi_{i} \lambda_{l}=0$ for every $i$. Consequently, $\lambda_{l}$ is constant on $\mathscr{W}_{x}^{\prime}$.

Thus we know that every principal curvature of $M$ is locally constant on an open and dense subset of $M$. Since $M$ is assumed to be connected, each principal curvature is constant on $M$. We therefore find by Berndt's classification that our real hypersurface $M$ in a quaternionic hyperbolic space is locally congruent to either a real hypersurface of type of $\left(\mathrm{H}_{1}\right),\left(\mathrm{H}_{2}\right)$ or $\left(\mathrm{H}_{3}\right)$. ["If" part of the proof of Theorems 3.1 and 3.2.]

In the second place we prove the if part. We shall prove each member of real hypersurfaces in the lists of Theorem A satisfies the condition $(*)$ case by case. At an arbitrary point $x \in M$ we choose orthonomal principal curvature vectors $v_{1}, \ldots, v_{4 n-4} \in \mathscr{D}_{x}$. We denote by $\lambda_{j}$ the principal curvature associtated with $v_{j}$ (i.e. $A v_{j}=\lambda_{j} v_{j}$ ) and by $\gamma_{j}$ the geodesic with initial vector $v_{j}$.

First, we study the case that $M$ is a real hypersurface of type $\left(\mathrm{P}_{1}\right),\left(\mathrm{H}_{1}\right)$ or $\left(\mathrm{H}_{3}\right)$ in a non-flat quaternionic space form. We shall show that $\dot{\gamma}_{j}$ is a principal curvature vector field along $\gamma_{j}$ for every $j$. It follows from Proposition 2.1(2) that

$$
\begin{aligned}
\nabla_{\dot{\gamma}_{j}}\left\|A \dot{\gamma}_{j}-\lambda_{j} \dot{\gamma}_{j}\right\|^{2} & =\nabla_{\dot{\gamma}_{j}}\left\langle A \dot{\gamma}_{j}, A \dot{\gamma}_{j}\right\rangle-2 \lambda_{j} \nabla_{\dot{\gamma}_{j}}\left\langle A \dot{\gamma}_{j}, \dot{\gamma}_{j}\right\rangle \\
& =2\left\langle\left(\nabla_{\dot{\gamma}_{j}} A\right) \dot{\gamma}_{j}, A \dot{\gamma}_{j}\right\rangle-2 \lambda_{j}\left\langle\left(\nabla_{\dot{\gamma}_{j}} A\right) \dot{\gamma}_{j}, \dot{\gamma}_{j}\right\rangle \\
& =\mp 2\left\langle\sum_{i=1}^{3}\left\{\left\langle\phi_{i} \dot{\gamma}_{j}, \dot{\gamma}_{j}\right\rangle \xi_{i}+\left\langle\xi_{i}, \dot{\gamma}_{j}\right\rangle \phi_{i} \dot{\gamma}_{j}\right\}, A \dot{\gamma}_{j}-\lambda_{j} \dot{\gamma}_{j}\right\rangle \\
& =\mp 2 \sum_{i=1}^{3}\left\langle\xi_{i}, \dot{\gamma}_{j}\right\rangle\left\langle\phi_{i} \dot{\gamma}_{j}, A \dot{\gamma}_{j}\right\rangle .
\end{aligned}
$$

Since $A \phi_{i}=\phi_{i} A$, we find $\left\langle\phi_{i} \dot{\gamma}_{j}, A \dot{\gamma}_{j}\right\rangle=\left\langle A \phi_{i} \dot{\gamma}_{j}, \dot{\gamma}_{j}\right\rangle=-\left\langle A \dot{\gamma}_{j}, \phi_{i} \dot{\gamma}_{j}\right\rangle$, which leads us to $\left\langle\phi_{i} \dot{\gamma}_{j}, A \dot{\gamma}_{j}\right\rangle=0$, and hence to $\nabla_{\dot{\gamma}_{j}}\left\|A \dot{\gamma}_{j}-\lambda_{j} \dot{\gamma}_{j}\right\|^{2}=0$. As $A \dot{\gamma}_{j}(0)=\lambda_{j} \dot{\gamma}_{j}(0)$, this shows that $A \dot{\gamma}_{j}(s)=\lambda_{j} \dot{\gamma}_{j}(s)$ for every $s$. By use of the formulae of Gauss and Weingarten, we can see that $\tilde{\nabla}_{\dot{\gamma}_{j}} \tilde{\nabla}_{\dot{\gamma}_{j}} \dot{\gamma}_{j}=\lambda_{j} \tilde{\nabla}_{\dot{\gamma}_{j}} \cdot \mathcal{N}=-\lambda_{j}^{2} \dot{\gamma}_{j}$, which means that the extrinsic shape of the geodesic $\gamma_{j}$ is a circle of curvature $\left|\lambda_{j}\right|$ in a non-flat quaternionic space form. 
Next we study the case that $M$ is a real hypersurface of type $\left(\mathrm{P}_{2}\right)$ in $\boldsymbol{H} P^{n}$. We decompose the tangent bundle $T M$ into the bundles of principal curvature vectors:

$$
T M=\mathscr{D} \oplus \mathscr{D}^{\perp}=\left(\mathscr{F}_{\lambda_{1}} \oplus \mathscr{F}_{\lambda_{2}}\right) \oplus\left(\mathscr{F}_{\mu_{1}} \oplus \mathscr{F}_{\mu_{2}}\right) .
$$

Here, when the ambient space is $\boldsymbol{H} P^{n}(4)$, the principal curvatures are $\lambda_{1}=\cot r$, $\lambda_{2}=-\tan r, \mu_{1}=2 \cot 2 r, \mu_{2}=-2 \tan 2 r$, and $\mathscr{F}_{v}$ denotes the subbundle of principal curvature vectors associated with $v$. For simplicity we choose canonical local basis of $\mathscr{J}$ on $\tilde{G}$ in Proposition B so that $\alpha_{1}=\mu_{1}$ and $\alpha_{2}=\alpha_{3}=\mu_{2}$. It follows from Proposition B and (2.2) that

$$
\left\{\begin{array}{llll}
\phi_{1}\left(\mathscr{F}_{\lambda_{1}}\right)=\mathscr{F}_{\lambda_{1}}, & \phi_{1}\left(\mathscr{F}_{\lambda_{2}}\right)=\mathscr{F}_{\lambda_{2}}, & \phi_{1}\left(\mathscr{F}_{\mu_{1}}\right)=\{0\}, & \phi_{1}\left(\mathscr{F}_{\mu_{2}}\right)=\mathscr{F}_{\mu_{2}} ; \\
\phi_{2}\left(\mathscr{F}_{\lambda_{1}}\right)=\mathscr{F}_{\lambda_{2}}, & \phi_{2}\left(\mathscr{F}_{\lambda_{2}}\right)=\mathscr{F}_{\lambda_{1}}, & \phi_{2}\left(\mathscr{F}_{\mu_{1}}\right) \subset \mathscr{F}_{\mu_{2}}, & \phi_{2}\left(\mathscr{F}_{\mu_{2}}\right)=\mathscr{F}_{\mu_{1}} ; \\
\phi_{3}\left(\mathscr{F}_{\lambda_{1}}\right)=\mathscr{F}_{\lambda_{2}}, & \phi_{3}\left(\mathscr{F}_{\lambda_{2}}\right)=\mathscr{F}_{\lambda_{1}}, & \phi_{3}\left(\mathscr{F}_{\mu_{1}}\right) \subset \mathscr{F}_{\mu_{2}}, & \phi_{3}\left(\mathscr{F}_{\mu_{2}}\right)=\mathscr{F}_{\mu_{1}} .
\end{array}\right.
$$

Since $\alpha_{1} \neq \alpha_{2}=\alpha_{3}$, again by using Proposition B we have $\left.q_{2}\right|_{\mathscr{D}_{x}}=\left.q_{3}\right|_{\mathscr{D}_{x}}=0$ and $q_{2}\left(\xi_{1}(x)\right)=q_{3}\left(\xi_{1}(x)\right)=0$ at each point $x \in G=\tilde{G} \cap M$.

We shall verify that both $\widetilde{F}_{\lambda_{1}} \oplus \boldsymbol{R} \xi_{1}$ and $\mathscr{F}_{\lambda_{2}} \oplus \boldsymbol{R} \xi_{1}$ over $G$ are integrable and moreover that each leaf of these local distributions is a totally geodesic submanifold of $M$. Since by (2.3) we have

$$
\nabla_{\xi_{1}} \xi_{1}=q_{3}\left(\xi_{1}\right) \xi_{2}-q_{2}\left(\xi_{1}\right) \xi_{3}+\phi_{1} A \xi_{1}=\alpha_{1} \phi_{1} \xi_{1}=0,
$$

we shall check that $\nabla_{X} \xi_{1}, \nabla_{\xi_{1}} X, \nabla_{X} Y$ are sections of $\mathscr{F}_{\lambda_{j}} \oplus \boldsymbol{R} \xi_{1}$ for all sections $X, Y$ of $\mathscr{F}_{\lambda_{j}}$. First, we see that

$$
\nabla_{X} \xi_{1}=q_{3}(X) \xi_{2}-q_{2}(X) \xi_{3}+\phi_{1} A X=\lambda_{j} \phi_{1} X
$$

hence $\nabla_{X} \xi_{1}$ is a section of $\mathscr{F}_{\lambda_{j}}$. Next we have

$$
\begin{aligned}
\left(\nabla_{\xi_{1}} A\right) X-\left(\nabla_{X} A\right) \xi_{1} & =\nabla_{\xi_{1}}(A X)-A \nabla_{\xi_{1}} X-\nabla_{X}\left(A \xi_{1}\right)+A \nabla_{X} \xi_{1} \\
& =\left(\lambda_{j} I-A\right) \nabla_{\xi_{1}} X-\lambda_{j}\left(\alpha_{1}-\lambda_{j}\right) \phi_{1} X \\
& =\left(\lambda_{j} I-A\right) \nabla_{\xi_{1}} X+\phi_{1} X .
\end{aligned}
$$

On the other hand, the Codazzi equation (2.5) leads us to

$$
\left(\nabla_{\xi_{1}} A\right) X-\left(\nabla_{X} A\right) \xi_{1}=\sum_{i=1}^{3}\left\{\left\langle\xi_{1}, \xi_{i}\right\rangle \phi_{i} X-\left\langle X, \xi_{i}\right\rangle \phi_{i} \xi_{1}-2\left\langle\phi_{i} \xi_{1}, X\right\rangle \xi_{i}\right\}=\phi_{1} X .
$$

Thus we obtain $\left(\lambda_{j} I-A\right) \nabla_{\xi_{1}} X=0$, hence $\nabla_{\xi_{1}} X$ is a section of $\mathscr{F}_{\lambda_{j}}$. Finally, we shall check $\nabla_{X} Y-\left\langle\nabla_{X} Y, \xi_{1}\right\rangle \xi_{1}$. We have

$$
\begin{aligned}
A\left(\nabla_{X} Y-\left\langle\nabla_{X} Y, \xi_{1}\right\rangle \xi_{1}\right)= & \nabla_{X}(A Y)-\left(\nabla_{X} A\right) Y-\alpha_{1}\left\langle\nabla_{X} Y, \xi_{1}\right\rangle \xi_{1} \\
= & \lambda_{j}\left(\nabla_{X} Y-\left\langle\nabla_{X} Y, \xi_{1}\right\rangle \xi_{1}\right) \\
& -\left(\nabla_{X} A\right) Y+\left(\lambda_{j}-\alpha_{1}\right)\left\langle\nabla_{X} Y, \xi_{1}\right\rangle \xi_{1} .
\end{aligned}
$$

Here we see that 


$$
\begin{aligned}
\left\langle\left(\nabla_{X} A\right) Y, \xi_{1}\right\rangle & =\left\langle Y,\left(\nabla_{X} A\right) \xi_{1}\right\rangle=\left\langle\nabla_{X}\left(A \xi_{1}\right)-A\left(\nabla_{X} \xi_{1}\right), Y\right\rangle \\
& =\left\langle\left(\alpha_{1} I-A\right) \nabla_{X} \xi_{1}, Y\right\rangle=\left\langle\nabla_{X} \xi_{1},\left(\alpha_{1} I-A\right) Y\right\rangle \\
& =\left(\alpha_{1}-\lambda_{j}\right)\left\langle\nabla_{X} \xi_{1}, Y\right\rangle=\left(\lambda_{j}-\alpha_{1}\right)\left\langle\nabla_{X} Y, \xi_{1}\right\rangle,
\end{aligned}
$$

hence $\left\langle-\left(\nabla_{X} A\right) Y+\left(\lambda_{j}-\alpha_{1}\right)\left\langle\nabla_{X} Y, \xi_{1}\right\rangle \xi_{1}, \xi_{1}\right\rangle=0$. For each section $Z$ which is orthogonal to $\xi_{1}$ we get by making use of (3.6)

$$
\begin{aligned}
\left\langle\left(\nabla_{X} A\right) Y, Z\right\rangle & =\left\langle Y,\left(\nabla_{X} A\right) Z\right\rangle \\
& =\left\langle\left(\nabla_{Z} A\right) X+\sum_{i=1}^{3}\left\{\left\langle X, \xi_{i}\right\rangle \phi_{i} Z-\left\langle Z, \xi_{i}\right\rangle \phi_{i} X-2\left\langle\phi_{i} X, Z\right\rangle \xi_{i}\right\}, Y\right\rangle \\
& =\left\langle\left(\nabla_{Z} A\right) X-\left\langle Z, \xi_{2}\right\rangle \phi_{2} X-\left\langle Z, \xi_{3}\right\rangle \phi_{3} X, Y\right\rangle \\
& =\left\langle\nabla_{Z}(A X)-A \nabla_{Z} X, Y\right\rangle \\
& =\left\langle\left(\lambda_{j} I-A\right) \nabla_{Z} X, Y\right\rangle \\
& =\left\langle\nabla_{Z} X,\left(\lambda_{j} I-A\right) Y\right\rangle=0 .
\end{aligned}
$$

Therefore we find that $\nabla_{X} Y-\left\langle\nabla_{X} Y, \xi_{1}\right\rangle \xi_{1}$ is a section of $\mathscr{F}_{\lambda_{j}}$, so that $\nabla_{X} Y$ is a section of $\mathscr{F}_{\lambda_{j}} \oplus \boldsymbol{R} \xi_{1}$. Consequently, the local distribution $\mathscr{F}_{\lambda_{j}} \oplus \boldsymbol{R} \xi_{1}$ is integrable and moreover each leaf $L$ of $\mathscr{F}_{\lambda_{j}} \oplus \boldsymbol{R} \xi_{1}$ is a totally geodesic submanifold of our real hypersurface $M$ for $j=1,2$. When $j=1$, the manifold $L$ is locally congruent to a geodesic sphere of radius $r$ in a complex projective space $C P^{n}(4)$ which is a totally complex totally geodesic submanifold of $\boldsymbol{H} P^{n}$, and when $j=2$, the manifold $L$ is locally congruent to a geodesic sphere of radius $\pi / 2-r$ in $C P^{n}(4)$.

We here recall the extrinsic shape of geodesics on a geodesic sphere in a complex projective space (see p. 540 of [MO]). Every geodesic $\gamma$ on a leaf $L$ of $\mathscr{F}_{\lambda_{j}} \oplus \boldsymbol{R} \xi_{1}$ with the initial vector $\dot{\gamma}(0) \in \mathscr{F}_{\lambda_{j}}$ is a circle of curvature $\left|\lambda_{j}\right|$ in $\boldsymbol{C} P^{n}$. Therefore, at each point $x \in M$ taking an orthonormal basis $\left\{v_{1}, \ldots, v_{2 n-2}\right\}$ of $\left(\mathscr{F}_{\lambda_{1}}\right)_{x}$ and an orthonormal basis $\left\{v_{2 n-1}, \ldots, v_{4 n-4}\right\}$ of $\left(\mathscr{F}_{\lambda_{2}}\right)_{x}$, we find that a real hypersurface of type $\left(\mathrm{P}_{2}\right)$ in a quaternionic projective space satisfies the condition $(*)$.

By just the same way as in the above discussion we can check that a real hypersurface of type $\left(\mathrm{H}_{2}\right)$ in a quaternionic hyperbolic space satisfies the condition $(*)$, and complete our proof for Theorems 3-1 and 3-2.

\section{Geodesics on geodesic spheres in a quaternionic space form}

In the preceding section, we characterized all curvature-adapted real hypersurfaces with constant principal curvatures in non-flat quaternionic space forms in terms of the extrinsic shape of geodesics of directions of principal curvature vectors. So, next it is natural to consider the extrinsic shape of all geodesics. In this context we devote the rest of this paper to study all geodesics on geodesic spheres in a quaternionic projective space, and on geodesic spheres, tubes around 
quaternionic hyperplane and horospheres in a quaternionic hyperbolic space. We shall start by observing an invariant for geodesics on these real hypersurfaces.

Let $M$ be a real hypersurface of type $\left(\mathrm{P}_{1}\right),\left(\mathrm{H}_{1}\right)$ or $\left(\mathrm{H}_{3}\right)$ in a non-flat quaternionic space form $\tilde{M}$. We denote by $\mathscr{N}$ a unit normal vector field on $M$ and put $\xi_{i}=-J_{i} \mathscr{N}(i=1,2,3)$ for an arbitrary local basis $\left\{J_{1}, J_{2}, J_{3}\right\}$ of the quaternionic structure on $\tilde{M}$. For a tangent vector $v \in T_{x} M$ we define nonnegative $\rho(v)$ by

$$
\rho(v)=\sqrt{\left\langle v, \xi_{1}\right\rangle^{2}+\left\langle v, \xi_{2}\right\rangle^{2}+\left\langle v, \xi_{3}\right\rangle^{2}},
$$

which is the norm of the projection of $v$ onto the space $\mathscr{D}_{x}^{\perp}$. We put $\rho_{\gamma}=\rho(\dot{\gamma})$ for a geodesic $\gamma$ on $M$. By using Weingarten formula and the equalities $A \phi_{i}=$ $\phi_{i} A(i=1,2,3)$ we find it is constant along $\gamma$ : Indeed,

$$
\begin{aligned}
\frac{1}{2} \frac{d}{d s} \rho_{\gamma}^{2}= & \left\langle\dot{\gamma}, q_{3}(\dot{\gamma}) \xi_{2}-q_{2}(\dot{\gamma}) \xi_{3}+\phi_{1} A \dot{\gamma}\right\rangle\left\langle\dot{\gamma}, \xi_{1}\right\rangle \\
& +\left\langle\dot{\gamma}, q_{1}(\dot{\gamma}) \xi_{3}-q_{3}(\dot{\gamma}) \xi_{1}+\phi_{2} A \dot{\gamma}\right\rangle\left\langle\dot{\gamma}, \xi_{2}\right\rangle \\
& +\left\langle\dot{\gamma}, q_{2}(\dot{\gamma}) \xi_{1}-q_{1}(\dot{\gamma}) \xi_{2}+\phi_{3} A \dot{\gamma}\right\rangle\left\langle\dot{\gamma}, \xi_{3}\right\rangle \\
= & \left\langle\dot{\gamma}, \phi_{1} A \dot{\gamma}\right\rangle\left\langle\dot{\gamma}, \xi_{1}\right\rangle+\left\langle\dot{\gamma}, \phi_{2} A \dot{\gamma}\right\rangle\left\langle\dot{\gamma}, \xi_{2}\right\rangle+\left\langle\dot{\gamma}, \phi_{3} A \dot{\gamma}\right\rangle\left\langle\dot{\gamma}, \xi_{3}\right\rangle=0 .
\end{aligned}
$$

We shall call this constant $\rho_{\gamma}$ (fibre) structure torsion of $\gamma$. Clearly it satisfies $0 \leqq \rho_{\gamma} \leqq 1$. When $M$ is one of a geodesic sphere in a quaternionic projective space, a geodesic sphere, a tube around quaternionic hyperplane and a horosphere in a quaternionic hyperbolic space, both of each vector $v \in \mathscr{D}$ and each vector $u \in \mathscr{D}^{\perp}$ are principal curvature vectors. So the structure torsion plays an important role in classifying geodesics on these real hypersurfaces.

First we study geodesics on a geodesic sphere $M=P_{1}^{0}(r)$ of radius $r$ in a quaternionic projective space $\boldsymbol{H} P^{n}(4)$ of quaternionic sectional curvature 4. Let $\tilde{\nabla}$ and $\nabla$ denote the Riemannian connections of $\boldsymbol{H} P^{n}(4)$ and $M$, respectively. For a geodesic $\gamma$ we also denote the curve $l \circ \gamma$ by $\gamma$ for simplicity. As a first step, we have

$$
\tilde{\nabla}_{\dot{\gamma}} \dot{\gamma}=\nabla_{\dot{\gamma}} \dot{\gamma}+\langle A \dot{\gamma}, \dot{\gamma}\rangle \mathscr{N}=\langle A \dot{\gamma}, \dot{\gamma}\rangle \mathscr{N} .
$$

This shows that the curve $\imath \circ \gamma$ is a geodesic on $\boldsymbol{H} P^{n}$ when $\langle A \dot{\gamma}, \dot{\gamma}\rangle=\cot r-$ $\rho_{\gamma}^{2} \tan r=0$. If it is not this case, we set as

$$
\begin{aligned}
& \kappa_{1}=|\langle A \dot{\gamma}, \dot{\gamma}\rangle|=\left|\cot r-\rho_{\gamma}^{2} \tan r\right|, \\
& X_{2}= \begin{cases}\mathscr{N} & \text { if }\langle A \dot{\gamma}, \dot{\gamma}\rangle>0, \\
-\mathscr{N} & \text { if }\langle A \dot{\gamma}, \dot{\gamma}\rangle<0 .\end{cases}
\end{aligned}
$$

Differentiating $X_{2}$, we have $\tilde{\nabla}_{\dot{\gamma}} X_{2}=-\kappa_{1} \dot{\gamma}+\kappa_{2} X_{3}$ with

$$
\kappa_{2}=\sqrt{\|A \dot{\gamma}\|^{2}-\langle A \dot{\gamma}, \dot{\gamma}\rangle^{2}}=\rho_{\gamma} \sqrt{1-\rho_{\gamma}^{2}} \cot r,
$$




$$
X_{3}= \begin{cases}\frac{1}{\kappa_{2}}(\langle A \dot{\gamma}, \dot{\gamma}\rangle \dot{\gamma}-A \dot{\gamma}) & \text { if }\langle A \dot{\gamma}, \dot{\gamma}\rangle>0, \\ -\frac{1}{\kappa_{2}}(\langle A \dot{\gamma}, \dot{\gamma}\rangle \dot{\gamma}-A \dot{\gamma}) & \text { if }\langle A \dot{\gamma}, \dot{\gamma}\rangle<0 .\end{cases}
$$

Thus, when $\rho_{\gamma}=0$ and $\rho_{\gamma}=1$, we see that the curve $\imath \circ \gamma$ is a circle of curvature $\cot r$ and $2|\cot 2 r|$, respectively.

In order to continue our calculation we set $\phi(v)=\sum_{i=1}^{3}\left\langle v, \xi_{i}\right\rangle \phi_{i}(v)$ for a tangent vector $v \in T M$, which does not depend on the choice of canonical local basis. One can easily compute that $\|\phi(v)\|^{2}=\rho^{2}(v)-\rho^{4}(v)$. By making use of (2.3) we obtain $\tilde{\nabla}_{\dot{\gamma}} X_{3}=-\kappa_{2} X_{2}+\kappa_{3} X_{4}$ and $\tilde{\nabla}_{\dot{\gamma}} X_{4}=-\kappa_{3} X_{3}$ with $\kappa_{3}=\cot r$ and

$$
X_{4}= \begin{cases}\frac{1}{\rho_{\gamma} \sqrt{1-\rho_{\gamma}^{2}}} \phi(\dot{\gamma}) & \text { if }\langle A \dot{\gamma}, \dot{\gamma}\rangle>0, \\ \frac{-1}{\rho_{\gamma} \sqrt{1-\rho_{\gamma}^{2}}} \phi(\dot{\gamma}) & \text { if }\langle A \dot{\gamma}, \dot{\gamma}\rangle<0 .\end{cases}
$$

Thus we get

Proposition 4.1. For each geodesic $\gamma$ on a geodesic sphere $P_{1}^{0}(r)$ of radius $r(0<r<\pi / 2)$ in $\boldsymbol{H} P^{n}(4)$, the curve $l \circ \gamma$ lies on a totally geodesic $\boldsymbol{C} P^{2}(4)$. Moreover, the extrinsic shape of $\gamma$ is as follows:

(1) Suppose the radius $r$ satisfies $\pi / 4 \leqq r<\pi / 2$. When $\rho_{r}=\cot r$, the curve $\imath \circ \gamma$ is a geodesic.

(2) When $r \neq \pi / 4$, the curve $\iota \circ \gamma$ is a circle of curvature $2|\cot 2 r|$ if $\rho_{\gamma}=1$. This circle lies on a totally geodesic $C P^{1}(4)$.

(3) If $\gamma$ has null structure torsion, the curve $l \circ \gamma$ is a circle of curvature $\cot r$. This circle lies on a totally geodesic $\boldsymbol{R} P^{2}(1)$.

(4) Generally, if $\rho_{\gamma} \neq 0,1$, then the curve $l \circ \gamma$ is a helix of proper order 4 whose curvatures are described as

$$
\kappa_{1}=\left|\cot r-\rho_{\gamma}^{2} \tan r\right|, \quad \kappa_{2}=\rho_{r} \sqrt{1-\rho_{\gamma}^{2}} \tan r, \quad \kappa_{3}=\cot r .
$$

Every geodesic $\gamma$ on $P_{1}^{0}(r)$ is a simple curve.

We call a smooth curve $\gamma$ closed if there exists $s_{0}(\neq 0)$ with $\gamma\left(s+s_{0}\right)=\gamma(s)$ for all $s$. The minimum positive $s_{0}$ with such a property is called the length of $\gamma$ and is denoted by length $(\gamma)$. When $\gamma$ is not closed we put length $(\gamma)=\infty$. As a direct consequence of this proposition we obtain the following with the aid of Theorem 2.5 in [AMY].

Proposition 4.2. Let $\gamma$ be a geodesic on a geodesic sphere $P_{1}^{0}(r)$ of radius $r(0<r<\pi / 2)$ in $\boldsymbol{H} P^{n}(4)$.

(1) If the structure torsion of $\gamma$ is 1 , then $\gamma$ is closed and its length is $\pi \sin 2 r$. 
(2) If $\gamma$ has null structure torsion, then $\gamma$ is also closed and its length is $2 \pi \sin r$. only if

(3) When the structure torsion $\rho_{\gamma}$ of $\gamma$ satisfies $0<\rho_{\gamma}<1$, it is closed if and

$$
\rho_{\gamma}=\frac{q}{\sin r \sqrt{p^{2} \tan ^{2} r+q^{2}}}
$$

with some relatively prime positive integers $p$ and $q$ with $q<p \tan ^{2} r$. In this case, its length is

$$
\text { length }(\gamma)= \begin{cases}2 \pi \sqrt{p^{2} \sin ^{2} r+q^{2} \cos ^{2} r}, & \text { if } p q \text { is even } \\ \pi \sqrt{p^{2} \sin ^{2} r+q^{2} \cos ^{2} r}, & \text { if } p q \text { is odd }\end{cases}
$$

We now make mention of the length spectrum of geodesic spheres in $\boldsymbol{H} \boldsymbol{P}^{n}$. We denote by $\operatorname{Geod}(N)$ the moduli space of geodesics on a Riemannian manifold $N$, which is the set of all congruency classes of geodesics on $N$ under the action of the isometry group of $N$. The length spectrum $\mathscr{L}_{N}: \operatorname{Geod}(N) \rightarrow$ $\boldsymbol{R} \cup\{\infty\}$ is defined by $\mathscr{L}_{N}([\gamma])=$ length $(\gamma)$. We also call the image $\operatorname{Lspec}(N)=$ $\mathscr{L}_{N}(\operatorname{Geod}(N)) \cap \boldsymbol{R}$ the length spectrum of $N$. For a length spectrum $\lambda \in \operatorname{Lspec}(N)$ we call the cardinality $m_{N}(\lambda)$ of the set $\mathscr{L}_{N}^{-1}(\lambda)$ the multiplicity of $\lambda$. When the multiplicity of a length spectrum is 1 we say it is simple. For congruency of geodesics on a geodesic sphere in $\boldsymbol{H} P^{n}$ we obtain the following.

Proposition 4.3. On a geodesic sphere $M$ in a quaternionic projective space, two geodesics are congruent with respect to the isometry group of $M$ if and only if their structure torsions coincide.

Proof. Let $\gamma_{1}$ and $\gamma_{2}$ be geodesics on $M$. Since the isometric embedding $l$ is equivariant, for each isometry $\varphi$ on $M$ there is an isometry $\tilde{\varphi}$ on $\boldsymbol{H} P^{n}$ with $\tilde{\varphi} \circ l=l \circ \varphi$. This implies that $l \circ \gamma_{1}$ and $l \circ \gamma_{2}$ are congruent if $\gamma_{1}$ and $\gamma_{2}$ are congruent. Comparing their curvatures we find $\rho_{\gamma_{1}}=\rho_{\gamma_{2}}$ if $\gamma_{1}$ and $\gamma_{2}$ are congruent.

Conversely, when $\gamma_{1}$ and $\gamma_{2}$ have the same structure torsion $\rho$, their initial vectors are of the form $\dot{\gamma}_{1}(0)=\sqrt{1-\rho^{2}} u_{1}+\rho \eta_{1}$ and $\dot{\gamma}_{2}(0)=\sqrt{1-\rho^{2}} u_{2}+\rho \eta_{2}$ with unit tangent vectors $u_{1}, u_{2} \in \mathscr{D}$ and $\eta_{1}, \eta_{2} \in \mathscr{D}^{\perp}$. By the following Lemma 4.4 we can choose an isometry $\varphi$ of $M$ with $d \varphi_{\gamma_{1}(0)}\left(u_{1}\right)=u_{2}$ and $d \varphi_{\gamma_{1}(0)}\left(\eta_{1}\right)=\eta_{2}$. Hence, $d \varphi_{\gamma_{1}(0)}\left(\dot{\gamma}_{1}(0)\right)=\dot{\gamma}_{2}(0)$, so that $\varphi \circ \gamma_{1}(s)=\gamma_{2}(s)$ for every $s$.

Lemma 4.4. Let $M=P_{1}^{0}(r)$ be a geodesic sphere in $\boldsymbol{H} P^{n}$. For any tangent vectors $u_{1}+\eta_{1} \in T_{x} M=\mathscr{D}_{x} \oplus \mathscr{D}_{x}^{\perp}$ and $u_{2}+\eta_{2} \in T_{y} M=\mathscr{D}_{y} \oplus \mathscr{D}_{y}^{\perp}$ of $M$ with $\left|u_{1}\right|=\left|u_{2}\right|=\left|\eta_{1}\right|=\left|\eta_{2}\right|=1$ at arbitrary points $x, y$, there exists an isometry $\tilde{\varphi}$ of $\boldsymbol{H} P^{n}$ with

i) $\tilde{\varphi}(M)=M$ and $\tilde{\varphi}(x)=y$,

ii) $d \tilde{\varphi}_{x}\left(u_{1}\right)=u_{2}, d \tilde{\varphi}_{x}\left(\eta_{1}\right)=\eta_{2}, d \tilde{\varphi}_{x}\left(\mathscr{N}_{x}\right)=\mathscr{N}_{y}$,

iii) $d \tilde{\varphi}_{x}\left(\mathscr{D}_{x}\right)=\mathscr{D}_{y}$ and $d \tilde{\varphi}_{x}\left(\mathscr{D}_{x}^{\perp}\right)=\mathscr{D}_{y}^{\perp}$. 
Proof. Let $\varpi: S^{4 n+3} \rightarrow \boldsymbol{H} P^{n}$ denote the $S^{3}$-fibration of a unit sphere $S^{4 n+3}$ in a right vector space $\boldsymbol{H}^{n+1}$. We decompose the tangent space $T_{w} S^{4 n+3}$ of $S^{4 n+3}$ at $w$ into the horizontal and vertical spaces: $T_{w} S^{4 n+3}=\mathscr{H}_{w} S^{4 n+3} \oplus \mathscr{V}_{w} S^{4 n+3}$. They are given by

$$
\begin{aligned}
\mathscr{H}_{w} S^{4 n+3} & =\left\{(w, v) \in\{w\} \times \boldsymbol{H}^{n+1} \mid \bar{w}_{0} v_{0}+\cdots+\bar{w}_{n} v_{n}=0\right\}, \\
\mathscr{V}_{w} S^{4 n+3} & =\left\{(w, w \lambda) \in\{w\} \times \boldsymbol{H}^{n+1} \mid \lambda \in \boldsymbol{H}, \operatorname{Re}(\lambda)=0\right\} .
\end{aligned}
$$

Here for $\lambda \in \boldsymbol{H}=\left\{a_{0}+a_{1} j_{1}+a_{2} j_{2}+a_{3} j_{3} \mid a_{i} \in \boldsymbol{R}\right\}$ we denote by $\bar{\lambda}$ its quaternionic conjugate and by $\operatorname{Re}(\lambda)$ its real part, which is given as $(\lambda+\bar{\lambda}) / 2$. Let $\hat{M}$ denote a hypersurface in $S^{4 n+3}$ given by

$$
\left\{w=\left.\left(w_{0}, w_{1}, \ldots, w_{n}\right) \in \boldsymbol{H}^{n+1}|| w_{0}|=\cos r,| w_{1}\right|^{2}+\cdots+\left|w_{n}\right|^{2}=\sin ^{2} r\right\},
$$

where $|\lambda|=(\bar{\lambda} \lambda)^{1 / 2}$. We see that $\varpi^{-1}(M)$ is isometric to $\hat{M}$.

For simplicity we only treat the case $n=2$ and $M=\varpi(\hat{M})$. At a point $w=\left(w_{0}, w_{1}, w_{2}\right) \in \hat{M}$ the tangent space of $\hat{M}$ is represented as

$$
T_{w} \hat{M}=\left\{(w, v) \in\{w\} \times \boldsymbol{H}^{3} \mid \operatorname{Re}\left(\bar{w}_{0} v_{0}\right)=\operatorname{Re}\left(\bar{w}_{1} v_{1}+\bar{w}_{2} v_{2}\right)=0\right\} .
$$

We denote by $\hat{\mathscr{N}}_{w} \in\left(T_{w} \hat{M}\right)^{\perp} \cap \mathscr{H}_{w} S^{11}$ the horizontal lift of the unit normal $\mathscr{N}$ at $\varpi(w)$, where $\left(T_{w} \hat{M}\right)^{\perp}$ denotes the orthogonal complement of $T_{w} \hat{M}$ in $T_{w} S^{11}$. When $w=\left(\cos r, w_{1}, w_{2}\right)$, it is of the form

$$
\hat{\mathscr{N}}_{w}=\left(w,\left(-\sin r, w_{1} \cot r, w_{2} \cot r\right)\right),
$$

hence the horizontal lifs of $\mathscr{D}_{\varpi(w)}$ and $\mathscr{D}_{\varpi(w)}^{\perp}$ are of the following forms:

$$
\begin{aligned}
& \hat{\mathscr{D}}_{w}=\left\{\left(w,\left(0, v_{1}, v_{2}\right)\right) \mid \bar{w}_{1} v_{1}+\bar{w}_{2} v_{2}=0\right\}, \\
& \hat{\mathscr{D}}_{w}^{\perp}=\left\{\left(w,\left(-\sin r, w_{1} \cot r, w_{2} \cot r\right) \cdot \lambda\right) \mid \operatorname{Re} \lambda=0\right\} .
\end{aligned}
$$

Put $z=(\cos r, \sin r, 0)(\in \hat{M})$. For $\left(w,\left(0, v_{1}, v_{2}\right)\right) \in \hat{\mathscr{D}}_{w}$ the othogonal matrix

$$
\left(\begin{array}{ccc}
1 & 0 & 0 \\
0 & w_{1}(\sin r)^{-1} & v_{1} \\
0 & w_{2}(\sin r)^{-1} & v_{2}
\end{array}\right) \in G L(3, \boldsymbol{H}) \cong G L(12, \boldsymbol{R})
$$

induces an isometry $\hat{\psi}$ of $S^{4 n+3}$ such that

i) $\hat{\psi}(z)=w$ and $\hat{\psi}(\hat{M})=\hat{M}$,

ii) $d \hat{\psi}_{z}\left(\hat{\mathscr{D}}_{z}\right)=\hat{\mathscr{D}}_{w}$ and $d \hat{\psi}_{z}\left(\hat{\mathscr{N}}_{z} \cdot \lambda\right)=\hat{\mathscr{N}}_{w} \cdot \lambda$ for every $\lambda \in \boldsymbol{H}$,

iii) $d \hat{\psi}_{z}((z,(0,0,1)))=\left(w,\left(0, v_{1}, v_{2}\right)\right)$.

Next for $v=a_{1} j_{1}+a_{2} j_{2}+a_{3} j_{3} \in \boldsymbol{H}$ with $|v|=1$ we choose an othogonal matrix

$$
A=\left(\begin{array}{lll}
-a_{1} & * & * \\
-a_{2} & * & * \\
-a_{3} & * & *
\end{array}\right) \in O(3)
$$


and consider an othogonal matrix

$$
\left(\begin{array}{lllll}
1 & & & & 0 \\
& A & & & \\
& & 1 & & \\
& & & A & \\
0 & & & & I_{4}
\end{array}\right) \in O(12),
$$

where $I_{4} \in G L(4, \boldsymbol{R})$ denotes the identity matrix. This induces an isometry $\hat{\Psi}$ of $S^{4 n+3}$ such that

i) $\hat{\Psi}(z)=z$ and $\hat{\Psi}(\hat{M})=\hat{M}$,

ii) $d \hat{\Psi}_{z}\left(\hat{\mathscr{D}}_{z}\right)=\hat{\mathscr{D}}_{z}$ and $d \hat{\Psi}_{z}\left(\hat{J}_{1} \hat{\mathscr{N}}_{z}\right)=\hat{\mathscr{N}}_{z} \cdot v$,

iii) $d \hat{\Psi}_{z}((z,(0,0,1)))=(z,(0,0,1))$,

where the real linear transformation $\hat{J}_{i}$ on $\boldsymbol{H}^{3}$ is defined by $\hat{J}_{i}(u)=u\left(-j_{i}\right)$. These guarantee the existence of an isometry $\tilde{\varphi}$ of $\boldsymbol{H} P^{n}$ with desirable conditions.

As a consequence of Proposition 4.2, for a geodesic sphere $M$ of radius $2 r / \sqrt{c}(0<r<\pi / 2)$ in $\boldsymbol{H} P^{n}(c)$, we see that its length spectrum coinsides with the length spectrum of a geodesic sphere of radius $2 r / \sqrt{c}$ in a complex projective space $C P^{n}(c)$ of holomorphic sectional curvature $c$ :

$$
\begin{aligned}
\operatorname{Lspec}(M) & =\left\{\frac{2 \pi}{\sqrt{c}} \sin 2 r\right\} \cup\left\{\frac{4 \pi}{\sqrt{c}} \sin r\right\} \\
& \cup\left\{4 \pi \sqrt{\frac{1}{c}\left(p^{2} \sin ^{2} r+q^{2} \cos ^{2} r\right)} \mid \begin{array}{l}
p \text { and } q \text { are relatively prime } \\
\text { positive integers which satisfy } \\
p q \text { is even and } q<p \tan ^{2} r
\end{array}\right\} \\
& \cup\left\{2 \pi \sqrt{\frac{1}{c}\left(p^{2} \sin ^{2} r+q^{2} \cos ^{2} r\right)} \mid \begin{array}{l}
p \text { and } q \text { are relatively prime } \\
\text { positive integers which satisfy } \\
p q \text { is odd and } q<p \tan ^{2} r
\end{array}\right\} .
\end{aligned}
$$

Therefore we obtain the following.

THEOREM 4.5. Let $M$ be a geodesic sphere of radius $2 r / \sqrt{c}(0<r<\pi / 2)$ in $\boldsymbol{H} P^{n}(c)$ of quaternionic sectional curvature $c$.

(1) The length spectrum $\operatorname{Lspec}(M)$ of $M$ is a discrete unbounded subset in the real line $\boldsymbol{R}$. In particular, there exist infinitely many congruency classes of closed geodesics.

(2) If $\tan ^{2} r$ is irrational, every length spectrum of $M$ is simple.

(3) If $\tan ^{2} r$ is rational, the multiplicity of each length spectrum of $M$ is finite. But it is not uniformly bounded; $\lim \sup _{\lambda \rightarrow \infty} m_{M}(\lambda)=\infty$. In this case, the growth order of $m_{M}$ is not so rapid. It satisfies $\lim _{\lambda \rightarrow \infty} \lambda^{-\delta} m_{M}(\lambda)=0$ for arbitrary positive $\delta$. 
(4) The cardinality $n_{M}(\lambda)$ of the set $\left\{[\gamma] \in \operatorname{Geod}(M) \mid \mathscr{L}_{M}([\gamma]) \leqq \lambda\right\}$ satisfies

$$
\lim _{\lambda \rightarrow \infty} \frac{n_{M}(\lambda)}{\lambda^{2}}=\frac{3 c r}{4 \pi^{4} \sin 2 r} .
$$

Next we study geodesics on a horosphere $H_{3}$, on a geodesic sphere $H_{1}^{0}(r)$ of radius $r$ and on a tube $H_{1}^{n-1}(r)$ of radius $r$ around quaternionic hyperplane in a quaternionic hyperbolic space $\boldsymbol{H} H^{n}(-4)$. Let $M$ be one of $H_{1}^{0}(r), H_{1}^{n-1}(r)$ and $\mathrm{H}_{3}$, and $\imath$ be an isometric embedding of $M$ into $\boldsymbol{H} H^{n}(-4)$. We denote by $\tilde{\nabla}$ the Riemannian connection of $\boldsymbol{H} H^{n}(-4)$. For each geodesic $\gamma$ on $M$ we find that $\imath \circ \gamma$ is a helix of order 4 in $\boldsymbol{H} H^{n}(-4)$ :

$$
\left\{\begin{aligned}
\tilde{\nabla}_{\dot{\gamma}} \dot{\gamma}= & \kappa_{1} X_{2}, \\
\tilde{\nabla}_{\dot{\gamma}} X_{2}= & -\kappa_{1} \dot{\gamma}+\kappa_{2} X_{3}, \\
\tilde{\nabla}_{\dot{\gamma}} X_{3}= & -\kappa_{2} X_{2}+\kappa_{3} X_{4}, \\
\tilde{\nabla}_{\dot{\gamma}} X_{4}= & -\kappa_{3} X_{3},
\end{aligned}\right.
$$

where

$$
\begin{aligned}
& \kappa_{1}=\langle A \dot{\gamma}(s), \dot{\gamma}(s)\rangle, \quad \kappa_{2}=\sqrt{\|A \dot{\gamma}\|^{2}-\langle A \dot{\gamma}, \dot{\gamma}\rangle^{2}}, \quad \kappa_{3}=\frac{1}{\kappa_{2}} \rho_{\gamma} \sqrt{1-\rho_{\gamma}^{2}}, \\
& X_{2}=\mathscr{N}, \quad X_{3}=\frac{1}{\kappa_{2}}(\langle A \dot{\gamma}, \dot{\gamma}\rangle \dot{\gamma}-A \dot{\gamma}), \quad X_{4}=\frac{1}{\rho_{\gamma} \sqrt{1-\rho_{\gamma}^{2}}} \sum_{i=1}^{3}\left\langle\dot{\gamma}, \xi_{i}\right\rangle \phi_{i}(\dot{\gamma})
\end{aligned}
$$

with a unit normal vector field $\mathscr{N}$ and the shape operator $A$ of $M$ in $\boldsymbol{H} H^{n}$. Thus we get the following result on the extrinsic shape of geodesics on those real hypersurfaces in $\boldsymbol{H} H^{n}$.

Proposition 4.6. Let $M$ be a real hypersurface in $\boldsymbol{H}^{n}(-4)$ which is one of a horosphere, a geodesic sphere of radius $r(0<r<\infty)$, and a tube of radius $r(0<r<\infty)$ around quaternionic hyperplane $\boldsymbol{H}^{n-1}$. For a geodesic $\gamma$ on $M$ the curve $l \circ \gamma$ lies on a totally geodesic $\mathrm{CH}^{2}(-4)$. Moreover, the extrinsic shape $\imath \circ \gamma$ is as follows:

(1) If $\rho_{\gamma}=1$, the curve $l \circ \gamma$ is a circle in $\boldsymbol{H} H^{n}$ which lies on a totally geodesic $\mathrm{CH}^{1}(-4)$. Its curvature is 2 if $M$ is a horosphere, 2 coth $2 r$ if $M$ is a geodesic sphere of radius $r$ or a tube of radius $r$ around quaternionic hyperplane.

(2) If $\rho_{\gamma}=0$, the curve $l \circ \gamma$ is also a circle which lies on a totally geodesic $\boldsymbol{R} H^{2}(-1)$. Its curvature is 1 if $M$ is a horosphere, coth $r$ if $M$ is a geodesic sphere of radius $r$, and $\tanh r$ if $M$ is a tube of radius $r$ around quaternionic hyperplane. 
(3) Generally, if $0<\rho_{\gamma}<1$, the curve $l \circ \gamma$ is a helix of proper order 4. Its curvatures are as the following table:

\begin{tabular}{|c|c|c|c|}
\hline & $H_{3}$ & $H_{1}^{0}(r)$ & $H_{1}^{n-1}(r)$ \\
\hline$\kappa_{1}$ & $1+\rho_{\gamma}^{2}$ & $\operatorname{coth} r+\rho_{\gamma}^{2} \tanh r$ & $\tanh r+\rho_{\gamma}^{2} \operatorname{coth} r$ \\
\hline$\kappa_{2}$ & $\rho_{\gamma} \sqrt{1-\rho_{\gamma}^{2}}$ & $\rho_{\gamma} \sqrt{1-\rho_{\gamma}^{2}} \tanh r$ & $\rho_{\gamma} \sqrt{1-\rho_{\gamma}^{2}} \operatorname{coth} r$ \\
\hline$\kappa_{3}$ & 1 & $\operatorname{coth} r$ & $\tanh r$ \\
\hline
\end{tabular}

Every geodesic on these manifolds is a simple curve.

As a consequence of this proposition we obtain the following (see [AMY]).

THEOREM 4.7. Let $\gamma$ be a geodesic on a geodesic sphere $M$ of radius $r(0<r<\infty)$ in $\boldsymbol{H} H^{n}(-4)$.

(1) When $\rho_{\gamma}=1$, it is closed with length $\pi \sinh 2 r$.

(2) When $\rho_{\gamma}=0$, it is also closed and its length is $2 \pi \sinh r$.

(3) When $0<\rho_{\gamma}<1$, it is closed if and only if

$$
\rho_{\gamma}=\frac{q}{\sinh r \sqrt{p^{2} \tanh ^{2} r-q^{2}}}
$$

with some relatively prime positive integers $p$ and $q$ with $q<p \tanh ^{2} r$. In this case, its length is

$$
\text { length }(\gamma)= \begin{cases}2 \pi \sqrt{p^{2} \sinh ^{2} r-q^{2} \cosh ^{2} r}, & \text { if } p q \text { is even } \\ \pi \sqrt{p^{2} \sinh ^{2} r-q^{2} \cosh ^{2} r}, & \text { if } p q \text { is odd }\end{cases}
$$

We call a smooth curve $\sigma$ on a quaternionic hyperbolic space $\boldsymbol{C} H^{n}$ unbounded in both directions if both $\sigma([0, \infty))$ and $\sigma((-\infty, 0])$ are unbounded sets. Considering the ideal boundary $\partial \boldsymbol{H} H^{n}$ of $\boldsymbol{H} H^{n}$ as a Hadamard manifold, we can define its limit points at infinity

$$
\sigma(\infty)=\lim _{t \rightarrow \infty} \sigma(t), \quad \sigma(-\infty)=\lim _{t \rightarrow-\infty} \sigma(t) \in \partial \boldsymbol{H} H^{n},
$$

if they exist. We shall call a smooth curve $\sigma$ on $\boldsymbol{H} H^{n}$ horocyclic if the following conditions hold.

i) It has single point at infinity; $\sigma(\infty)=\sigma(-\infty)$.

ii) If a geodesic $\rho$ on $\boldsymbol{H} H^{n}$ with $\rho(\infty)=\sigma(\infty)$ crosses $\sigma$ at some point $\rho\left(s_{0}\right)$, then they cross orthogonally at $\rho\left(s_{0}\right)$. 
These conditions are equivalent to the condition that $\sigma$ is unbounded in both directions and lies on a horosphere. We obtain the following along the same lines as in $[\mathrm{AMY}]$.

THEOREM 4.8. Let $\gamma$ be a geodesic on a tube $M$ of radius $r(0<r<\infty)$ around quaternionic hyperplane $\boldsymbol{H} H^{n-1}$ in $\boldsymbol{H}^{n}(-4)$.

(1) When $\rho_{\gamma}=1$, it is closed and its length is $\pi \sinh 2 r$.

(2) When $\rho_{\gamma}<1 / \cosh r$, it is unbounded in both directions, and has two distinct points at infinity as a curve on $\boldsymbol{H} H^{n}$.

(3) When $\rho_{\gamma}=1 / \cosh r$, it is horocyclic as a curve on $\boldsymbol{H} H^{n}$.

(4) When $1 / \cosh r<\rho_{\gamma}<1$, it is bounded. Under this situation, it is closed if and only if

$$
\rho_{\gamma}=\frac{p}{\cosh r \sqrt{p^{2}-q^{2} \operatorname{coth}^{2} r}}
$$

with some relatively prime positive integers $p$ and $q$ with $p \tanh ^{2} r>q$. In this case, its length is

$$
\text { length }(\gamma)= \begin{cases}2 \pi \sqrt{p^{2} \sinh ^{2} r-q^{2} \cosh ^{2} r}, & \text { if } p q \text { is even } \\ \pi \sqrt{p^{2} \sinh ^{2} r-q^{2} \cosh ^{2} r}, & \text { if } p q \text { is odd }\end{cases}
$$

Proposition 4.9. Every geodesic on a horosphere in a quaternionic hyperbolic space is unbounded in both directions, hence it is horocyclic as a curve on a quaternionic hyperbolic space.

In order to make mention of length spectrum we need a congruence theorem. We set

$$
H_{1}^{4 n+3}=\left\{w=\left.\left(w_{0}, \ldots, w_{n}\right) \in \boldsymbol{H}^{n+1}|-| w_{0}\right|^{2}+\left|w_{1}\right|^{2}+\cdots\left|w_{n}\right|^{2}=-1\right\}
$$

and denote by $\varpi: H_{1}^{4 n+3} \rightarrow \boldsymbol{H} H^{n}$ the $S^{3}$-fibration. We consider the following hypersurfaces in $H_{1}^{4 n+3}$ :

$$
\begin{aligned}
& \hat{M}_{1}=\left\{w \in \boldsymbol{H}^{n+1}\left|w_{0}\right|=\cosh r,\left|w_{1}\right|^{2}+\cdots+\left|w_{n}\right|^{2}=\sinh ^{2} r\right\}, \\
& \hat{M}_{2}=\left\{w \in \boldsymbol{H}^{n+1} \mid \begin{array}{c}
-\left|w_{0}\right|^{2}+\left|w_{1}\right|^{2}+\cdots+\left|w_{n-1}\right|^{2}=-\cosh ^{2} r, \\
\left|w_{n}\right|=\sinh r
\end{array}\right\}, \\
& \hat{M}_{3}=\left\{w \in \boldsymbol{H}^{n+1} \mid \begin{array}{c}
-\left|w_{0}\right|^{2}+\left|w_{1}\right|^{2}+\cdots+\left|w_{n}\right|^{2}=-1, \\
\left|w_{0}-w_{1}\right|=1
\end{array}\right\} .
\end{aligned}
$$

According as $M$ is a geodesic sphere of radius $r$, a tube of radius $r$ around quaternionic hyperplane or a horosphere in $\boldsymbol{H} H^{n}(-4)$, the manifold $\varpi^{-1}(M)$ is isometric to $\hat{M}_{1}, \hat{M}_{2}$ or $\hat{M}_{3}$. By a similar argument to that in the proof of Lemma 4.4 and Proposition 4.3 we obtain the following. 
Proposition 4.10. Let $M$ be one of a horosphere, a geodesic sphere and a tube around quaternionic hyperplane in a quaternionic hyperbolic space. Two geodesics on $M$ are congruent with respect to the isometry group of $M$ if and only if their structure torsions coincide.

With the aid of this propsition, Theorem 4.7 assures that the length spectrum of a geodesic sphere $M$ of radius $2 r / \sqrt{c}$ in $\boldsymbol{H} H^{n}(-c)$ is of the following form;

$$
\begin{aligned}
& \operatorname{Lspec}(M)=\left\{\frac{4 \pi}{\sqrt{c}} \sinh r\right\} \cup\left\{\frac{2 \pi}{\sqrt{c}} \sinh 2 r\right\} \\
& \cup\left\{\begin{array}{l|l}
4 \pi \sqrt{\frac{1}{c}\left(p^{2} \sinh ^{2} r-q^{2} \cosh ^{2} r\right)} & \begin{array}{l}
p \text { and } q \text { are relatively prime } \\
\text { positive integers which satisfy } \\
p q \text { is even and } q<p \tanh ^{2} r
\end{array}
\end{array}\right\} \\
& \cup\left\{\begin{array}{l|l}
2 \pi \sqrt{\frac{1}{c}\left(p^{2} \sinh ^{2} r-q^{2} \cosh ^{2} r\right)} \mid \begin{array}{c}
p \text { and } q \text { are relatively prime } \\
\text { positive integers which satisfy } \\
p q \text { is odd and } q<p \tanh ^{2} r
\end{array}
\end{array}\right\},
\end{aligned}
$$

and Theorem 4.8 assures that the length spectrum of a tube $M$ of radius $2 r / \sqrt{c}$ around quaternionic hyperplane $\boldsymbol{H} H^{n-1}$ in $\boldsymbol{H} H^{n}(-c)$ is of the following form;

$$
\begin{aligned}
& \operatorname{Lspec}(M)=\left\{\frac{2 \pi}{\sqrt{c}} \sinh 2 r\right\} \\
& \cup\left\{\begin{array}{l|l}
4 \pi \sqrt{\frac{1}{c}\left(p^{2} \sinh ^{2} r-q^{2} \cosh ^{2} r\right)} & \begin{array}{l}
p \text { and } q \text { are relatively prime } \\
\text { positive integers which satisfy } \\
p q \text { is even and } q<p \tanh ^{2} r
\end{array}
\end{array}\right\} \\
& \cup\left\{\begin{array}{l|l}
2 \pi \sqrt{\frac{1}{c}\left(p^{2} \sinh ^{2} r-q^{2} \cosh ^{2} r\right)} & \begin{array}{c}
p \text { and } q \text { are relatively prime } \\
\text { positive integers which satisfy } \\
p q \text { is odd and } q<p \tanh ^{2} r
\end{array}
\end{array}\right\} .
\end{aligned}
$$

We obtain the following on the multiplicity of the length spectrum.

THEOREM 4.11. Let $M$ be either a geodesic sphere of radius $2 r / \sqrt{c}$ or a tube of radius $2 r / \sqrt{c}$ around quaternionic hyperplane $\boldsymbol{H} H^{n-1}$ in $\boldsymbol{H} H^{n}(-c)$ of quaternionic sectional curvature $-c$.

(1) The length spectrum $\operatorname{Lspec}(M)$ is a discrete unbounded subset of the real line $\boldsymbol{R}$. In particular, there exist infinitely many congruency classes of closed geodesics.

(2) If $\operatorname{coth}^{2} r$ is irrational of the form either

$$
\frac{1}{2 q^{2}}\left\{\left(p^{2}+q^{2}-1\right)-\sqrt{\left\{p^{2}-(q-1)^{2}\right\}\left\{p^{2}-(q+1)^{2}\right\}}\right\}
$$


for some relatively prime positive integers $p, q$ with even $p q$ and $p \geq q+3$, or

$$
\frac{1}{2 q^{2}}\left\{\left(p^{2}+q^{2}-4\right)-\sqrt{\left\{p^{2}-(q-2)^{2}\right\}\left\{p^{2}-(q+2)^{2}\right\}}\right\}
$$

for some relatively prime positive integers $p, q$ with odd $p q$ and $p \geq q+4$, then the multiplicity of the spectrum $(2 \pi / \sqrt{c})$ sinh $2 r$ is two and other length spectrum of $M$ is simple.

(3) If $\operatorname{coth}^{2} r$ is irrational and is not of the form in (2), then every length spectrum of $M$ is simple.

(4) If $\operatorname{coth}^{2} r$ is rational, the multiplicity of each length spectrum is finite. But it is not uniformly bounded; $\lim _{\sup _{\lambda \rightarrow \infty}} m_{M}(\lambda)=\infty$. The growth order of $m_{M}(\lambda)$ is not so rapid. It satisfies $\lim _{\lambda \rightarrow \infty} \lambda^{-\delta} m_{M}(\lambda)=0$ for arbitrary positive $\delta$.

(5) The number $n_{M}(\lambda)$ of congruency classes of geodesics on $M$ with length not greater than $\lambda$ satisfies

$$
\lim _{\lambda \rightarrow \infty} \frac{n_{M}(\lambda)}{\lambda^{2}}=\frac{3 c r}{4 \pi^{4} \sinh 2 r}
$$

We here go back to the viewpoint of characterizations of curvature-adapted real hypersurfaces with constant principal curvatures by the extrinsic shape of geodesics. By Propositions 4.1 and 4.6 and the same argument as in the proof of Propositions 4.1 and 4.2 in [AMY], we see the following.

Proposition 4.12. A real hypersurface $M$ in a non-flat quaternionic space form $\tilde{M}$ of quaternionic dimension $n(\geqq 3)$ is locally congruent to one of a geodesic sphere, a tube around quaternionic hyperplane and a horosphere if and only if at each point $x \in M$ there exists an orthonormal basis $\left\{v_{1}, v_{2}, \ldots, v_{4 n-4}\right\}$ of $\mathscr{D}_{x}$ $\left(\subset T_{x} M\right)$ such that all geodesics on $M$ emanating $x$ in the direction $v_{j}+v_{k}$ $(1 \leqq j \leqq k \leqq 4 n-4)$ are circles of positive curvature in $\tilde{M}$.

Proposition 4.13. A real hypersurface $M$ in $\boldsymbol{H} H^{n}(-c)(n \geqq 2)$ is locally congruent to a horosphere if and only if at each point $x \in M$ there exists an orthonormal basis $\left\{v_{1}, v_{2}, \ldots, v_{4 n-4}\right\}$ of $\mathscr{D}_{x}$ such that all geodesics on $M$ with the initial vector $v_{j}(1 \leqq j \leqq 4 n-4)$ are circles of curvature $\sqrt{c} / 2$ on $\boldsymbol{H} H^{n}(-c)$.

\section{REFERENCES}

[A] T. AdACHI, Circles on quaternionic space forms, J. Math. Soc. Japan, 48 (1996), 205-227.

[AKM] T. AdAchi, M. KimuRA AND S. MAEDA, A characterization of all homogeneous real hypersurfaces in a complex projective space by observing the extrinsic shape of geodesics, Arch. Math. (Basel), 73 (1999), 303-310.

[AMY] T. Adachi, S. Maeda AND M. Yamagishi, Length spectrum of geodesic spheres in a nonflat complex space form, to appear in J. Math. Soc. Japan.

[B] J. B. BERNDT, Real hypersurfaces in quaternionic space forms, J. reine angew. Math., 419 (1991), 9-26. 
[CM] B.-Y. CHEN AND S. MAEDA, Hopf hypersurfaces with constant principal curvatures in complex projective or complex hyperbolic spaces, to appear in Tokyo J. Math..

[H] T. Hamada, On real hypersurfaces of a quaternionic projective space, Saitama Math. J., 11 (1993), 29-39.

[MO] S. Maeda And K. Ogiue, Characterizations of geodesic hyperspheres in a complex projective space by observing the extrinsic shape of geodesics, Math. Z., 225 (1997), 537-542.

[MP] A. Martínez and J. D. PÉREZ, Real hypersurfaces in quaternionic projective space, Ann. Math. Pure Appl., 145 (1986), 355-384.

[NR] R. Niebergall and P. J. Ryan, Real hypersurfaces in complex space forms, Tight and Taut Submanifolds, (T. E. Cecil and S. S. Chern eds.), Cambridge University Press, 1998, 233-305.

[P] J. S. PAK, Real hypersurfaces in quaternionic kaehlerian manifolds with constant $Q$ sectional curvature, Kodai Math. Sem. Rep., 29 (1977), 22-61.

Department of Mathematics

Nagoya Institute of Technology

GOKISO, NAGOYA, 466-8555, JAPAN

e-mail: adachi@math.kyy.nitech.ac.jp

Department of Mathematics

SHIMANE UNIVERSITY

Matsue, Shimane, 690-8504, JAPAN

e-mail: smaeda@math.shimane-u.ac.jp 